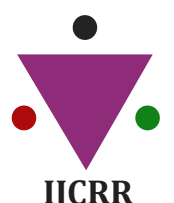

IJCRR

Section: Healthcare

Sci. Journal Impact

Factor: 6.1 (2018)

ICV: 90.90 (2018)

(c) (i) (3)

Copyright@IJCRR

\section{Lipid-Based Solid Dispersions of Azilsartan Medoxomil with Improved Oral Bioavailability: In Vitro and In Vivo Evaluation}

\section{Dhiraj Kumar Chopra, Durga Madhab Kar, Pratap Kumar Sahu}

School of Pharmaceutical Sciences, Siksha 'O' Anusandhan (Deemed to be University), Kalinga Nagar, Ghatikia, Bhubaneswar-751003, Orissa, India.

\title{
ABSTRACT
}

Introduction: Among the various techniques established, lipid-based solid dispersion approach is one by which the bioavailability of BCS class II drug shall be significantly increased without affecting the permeability of drug.

Aim \& Objective: The main aim of the current research work is to evaluate the potential of lipid-based solid dispersions of Azilsartan Medoxomil to enhance oral bioavailability of Azilsartan Medoxomil (BCS Class IV molecule).

Methodology: ASD has been prepared using different hydrophilic lipid-based carriers such as gelucire 44/14 and gelucire 50/13 at the ratio of $1: 1,1: 2$ and 1:3. Pearlitol SD 200 was used as the hydrophilic carrier in all the formulations to convert the lipidbased solid dispersion to free-flowing powder. Solid dispersions were prepared by the solvent evaporation method. Prepared ASD's were evaluated for their micromeritics properties, saturation solubility, in vitro dissolution, and in vivo bioavailability in selected rats.

Results: Among the formulations prepared, formulation (ASD6) containing gelucire 50/13 (1:3), Pearlitol SD 200 as the carrier has shown enhanced drug release $(98.9 \pm 1.9$ release in 30 minutes) and solubility $(65.57 \mathrm{mg} / \mathrm{mL}) \mathrm{compared} \mathrm{to} \mathrm{other} \mathrm{formu-}$ lations. Hence, this formulation is evaluated for comparative in vivo bioavailability in rats along with pure drug and marketed formulation (Zilarbi). It was found that relative bioavailability of ASD6 was increased by 1.11 times compared to pure drug and increased by 1.04 times compared to marketed formulation.

Conclusion: Hence, the study demonstrated that lipid-based solid dispersion technology can lead to improve the bioavailability of poorly soluble drugs like Azilsartan medoxomil significantly.

Key Words: Lipid-based, Liquid solid dispersions, Bioavailability, Dissolution, Solubility

\section{INTRODUCTION}

Till the date for the majority of the therapeutically active agents, the oral route is the major route of drug administration. Intrinsic solubility and rate of dissolution of the drug are very critical and important critical parameters for the drug oral absorption, especially for poorly soluble or insoluble drugs. Similarly, the permeability of the drug across the gastrointestinal tract is also crucial especially for highly hydrophilic drugs. Hence, dissolution is the rate-limiting step for highly lipophilic drugs and permeation is the rate-limiting steps for highly hydrophilic drugs in the process of their absorption from the gastrointestinal tract when they have administered through oral route. Due to poor solubility/limited dissolution rate and poor/limited permeability, class II and or class IV drugs suffer less bioavailability thereby decreased therapeutic effect. Several techniques have been reported to improve the solubility and dissolution properties of poorly soluble drugs which intern can improve absorption and bioavailability. ${ }^{1,2}$.

Among the various techniques established, solid dispersion approach is one by which the bioavailability of BCS class II drug shall be significantly increased. This technique is the most promising approach and has been proved to improve the bioavailability of poorly soluble drugs by several research scientists ${ }^{3,4,5}$. It was also reported that lipid-based solid dispersion shall be prepared with using lipid-based hydrophilic carriers by which the bioavailability of class II drugs have been increased by improving the drug dissolution

\section{Corresponding Author:}

Dhiraj Kumar Chopra, School of Pharmaceutical Sciences, Siksha 'O' Anusandhan (Deemed to be University), Kalinga Nagar, Ghatikia, Bhubaneswar-751003, Orissa; Email: dhirajchopraphd@gmail.com

ISSN: 2231-2196 (Print)

Received: 22.06 .2020
ISSN: 0975-5241 (Online)

Revised: 14.08 .2020
Accepted: 18.09 .2020 
rate and bioavailability of class IV drugs have been increased by improving both the dissolution and permeability. ${ }^{6-10}$

Azilsartan Medoxomil, novel prodrug, is a selective angiotensin II receptor blocker that is approved in the treatment of hypertension. As it is a prodrug, it undergoes rapid deesterification in the GIT before its absorption and produces its active form Azilsartan. Though it is administered as a prodrug, still the drug is having very less oral bioavailability in healthy humans as the drug belongs to BCS class IV and due to unfavourable breakage of the ester drug to a poorly permeable parent molecule in the gastrointestinal fluids. ${ }^{11-15}$

Hence, the objective of the present work was to enhance the solubility, dissolution rate and permeability and hence the oral bioavailability of poorly soluble BCS Class IV drug, Azilsartan medoxomil using lipid based solid dispersion approach.

\section{MATERIALS AND METHODS}

\section{Materials}

Azilsartan medoxomil gift sample was provided by Alembic Pharma Ltd,, Baroda, India. All the other materials were received as samples from Abitec Corp., USA.: Gelucire 44/14 and Gelucire 50/13 were received as gift samples from Gattefosse. Pearlitol SD 200, were received from Signet, India.

\section{Saturation solubility studies}

Saturation solubility studies of Azilsartan medoxomil was done in various buffers and non-volatile liquid vehicles. Excess amount of drug was added to two $\mathrm{mL}$ of each solvent in a screw-capped vial and was kept on the isothermal mechanical shaker at $25^{\circ} \mathrm{C}$ for 72 hours. After equilibrium/ saturation, each solution was centrifuged at $5000 \mathrm{rpm}$ for 30 minutes. The supernatant was collected and it was filtered through $0.45 \mu$ membrane filter, suitably diluted with methanol and then absorbance was measured at 249nm using UV spectrophotometer.

Phase solubility studies: Phase solubility studies were done by taking different concentrations of Gelucire 44/14 and Gelucire 50/13 $(1 \%, 3 \%, 5 \%$, and $7 \%)$ in distilled water. To each of these concentrations, the excess amount of drug was added. Then these solutions were kept for shaking on shaker for 48 hours. After 48 hours samples were filtered through the Whatman filter paper then the solution diluted and estimated for Azilsartan concentration using UV-spectroscopy. Three determinations were carried out for each sample to calculate the solubility.

\section{Preparation of Lipid-Based Solid Dispersions}

Lipid-based solid dispersions of Azilsartan were prepared by using different hydrophilic lipid-based carriers such as gelucire
44/14 and gelucire 50/13 in different ratios such as 1:1,1:2 and 1:3. These ratios were decided based on the results obtained in phase solubility studies. Pearlitol SD 200 was used as inert carrier in all the formulations. Compositions of various formulations are given in Table 1. Lipid-based solid dispersions (LBSD) of Azilsartan were prepared by the solvent evaporation method. Azilaratn $40 \mathrm{mg}$ was taken and dissolved in $10 \mathrm{ml}$ solvent mixture of ethanol and dichloromethane. To the drug required quantity of lipid-based carrier and the porous inert carrier was added. This solution was taken into the round bottom flask, attached to the rotary flash evaporator and evaporated at $37^{\circ} \mathrm{C}$, rpm was 60 for $15 \mathrm{~min}$. Solid dispersions were obtained, collected and dried in the desiccator till it was completely dried.

\section{Evaluation of formulations}

Flowability of prepared formulations was detected by Carr's index, angle of repose, and Hausner's ratio.

\section{Angle of Repose}

Angle of repose of all the prepared formulations were detected by using below provided formula:

$$
\theta=\operatorname{Tan}^{-1}(\mathrm{~h} / \mathrm{r})
$$

Where ' $h$ ' and ' $r$ ' are the height and radius of powder cone.

\section{Compressibility Index}

Carr's Compressibility index was calculated using below mentioned formula:

$$
\begin{aligned}
& \text { Carr' s index }(\%) \\
& \quad=\frac{[(\text { Tapped density }- \text { Bulk density }) \times 100]}{\text { Tapped density }} .
\end{aligned}
$$

\section{Hausner's Ratio}

Hausner's ratio was calculated from the equation:

Hausner's Ratio = Tapped Density/Bulk Desnity

\section{Content Uniformity}

Weight equivalent $(200 \mathrm{mg}$ ) to one unit dose of Azilsartn medoxomil (40mg) of each prepared formulation was transferred to $100 \mathrm{~mL}$ volumetric flask, $10 \mathrm{~mL}$ of methanol was added and shaken for $10 \mathrm{~min}$ and volume was made to $100 \mathrm{~mL}$ with $6.8 \mathrm{pH}$ buffer. The solution was filtered, diluted suitably, and analyzed spectrophotometrically using UV-visible double-beam spectrophotometer (UV1800, Shimadzu, Japan).

\section{Saturation Solubility Studies}

Saturation solubility studies of all the prepared formulations were done in water using the same method as mentioned above 


\section{In-Vitro Drug Release Study}

In vitro dissolution studies of all the prepared formulations were done using USP Type-II dissolution Paddle apparatus using $6.8 \mathrm{pH}$ Phosphate buffer as dissolution medium at $50 \mathrm{rpm}$ at $37^{\circ} \mathrm{C} \pm 0.5^{\circ} \mathrm{C}$. At each predetermined time intervals, $10 \mathrm{~mL}$ samples were withdrawn and replaced with fresh dissolution media. Upon filtration, through a $0.45 \mu \mathrm{m}$ membrane filter and suitable dilution each sample was analysed using UV spectrophotometer.

\section{In Vivo Pharmacokinetic Studies}

The research protocol of animal experimentation was approved by the Institutional Animal Ethics Committee (IAEC Number: 516/01/A/CPCSEA). The pharmacokinetic evaluation was done in healthy Wistar rats. The animals were housed in metallic cages with free access move and provided standard laboratory diet and water in central animal house. Based on the evaluation results of in vitro dissolution studies ASD3 that showed highest drug release within 30 minutes was selected to carry out in vivo performance in comparison with the pure drug solution (APD) and marketed formulation Zilarbi tablets. The animal dose was calculated using the following formula.

AnimalEquivalentDose $(\mathrm{mg} / \mathrm{kg})$

$=$ HumanDose $(\mathrm{mg} / \mathrm{kg})$ XKmvalueofanimal $/$ Kmvalueof $\mathrm{human}$

Km value of Rat is 7

$\mathrm{Km}$ value of Human is 37

As per the above formula dose of each formulation administered was $3.5 \mathrm{mg}$. Each formulation was prepared by taking the weight equivalent to $3.5 \mathrm{mg}$ of drug and dispersing in $1 \%$ Sodium CMC Suspension. All formulations were administered to animal through the oral cannula.

An open-label, balanced, randomized, three-period, threetreatment, three-sequence, single-dose crossover study design in which six healthy Wister rats received one treatment (product) each with a washout period of 7 days such that all products are tested in all the six healthy rats during the study. Six healthy rats with a bodyweight range of $200-250 \mathrm{~g}$ were selected through physical examination. Animals were randomly divided into three groups consisting of two in each group. Each group on three different periods received a single dose of each of the above three treatments in random order with a washout period of one week between each treatment and the scheme of administration of the treatments is shown below table 2 .

The animals were fasted overnight before administering the dose. After collecting the zero hour blood sample (blank), a standardized diet was given in the morning.

During each period, $0.5 \mathrm{~mL}$ venous blood samples were collected from the retro-orbital vein of each animal in Ac-
Cuvet tubes (Quantum Biologicals Pvt. Ltd., Chennai, India) containing K3EDTA. Blood Samples were collected at $0,0.5,1,1.5,2,3,4,6,8,10,1218$ and 24 hrs. Plasma was immediately separated by centrifugation at $5000 \mathrm{rpm}$ for $30 \mathrm{~min}$ from the blood samples and stored in frozen conditions at $-20^{\circ} \mathrm{C}$ with appropriate labelling of the subject code number, study date and collection time before analysis. The concentration of Azilsartan in plasma samples was measured by the HPLC method using Waters symmetry $\mathrm{C} 18(4.6 \times 250 \mathrm{~mm}, 5 \mu \mathrm{m})$ column as column, $25 \mathrm{mM}$ ammonium acetate buffer ( $\mathrm{pH} 5.5)$ : acetonitrile $55: 45 \mathrm{v} / \mathrm{v}$ as isocratic mobile phase and UV detector at 254nm. Pharmacokinetic parameters such as peak plasma concentration (Cmax), time at which Cmax occurred (Tmax), area under the curve (AUC), elimination rate constant (Kel), and biological half-life $\left(\mathrm{t}^{1} \frac{1}{2}\right)$ were calculated in each case using the data by Kinetics Pro 2.0 (PK Solver 2.0) software using the non-compartmental approach. Percent relative bioavailability of the optimized formulation vs pure drug suspension and the marketed formulation was also estimated to understand the improvement of oral bioavailability with the selected method.

\section{RESULTS AND DISCUSSION}

\section{Saturation Solubility Studies}

Solubility data of drug Azilsartan medoxomil in various liquid vehicles is shown in Figure 1. Azilsartan appears to be more soluble in alkaline $\mathrm{pH}$ than acidic $\mathrm{pH}$.

\section{Phase Solubility Studies}

Phase solubility studies were performed in different concentrations of gelucire 44/14 and 50/13 and the values obtained were represented in bar chart which is shown in Figures 2. It was observed that the phase solubility of the drug is increased with the increased concentration of the carrier.

\section{Preparation of Lipid-Based Solid Dispersions.}

Lipid-based solid dispersions of Azilsartan were prepared by using different hydrophilic lipid-based carriers such as gelucire 44/14 and gelucire 50/13 in different ratios such as $1: 1,1: 2$ and $1: 3$. These ratios were decided based on the results obtained in phase solubility studies. Pearlitol SD 200 was used as inert carrier in all the formulations. Compositions of various formulations are given in Table 1

\section{Evaluation of Formulations}

\section{Micromeritics Parameters}

Good flow is required and is crucial for content uniformity. The results of various flow parameters are shown in Table 3. 
All the formulations have shown good and improved flow compared to pure drug. This could be probably due to the presence of pearlitol.

\section{Content Uniformity}

All the prepared formulations were found to uniform in their drug content

\section{Saturation Solubility studies}

Results of saturation solubility studies are shown in figure 3. It was observed that all the prepared formulations have shown improved solubility in water and the formulation prepared with gelucire 50/13 carrier at 1:3 ratio (ASD6) has a comparatively higher solubility than other formulations.

\section{In Vitro Dissolution Studies}

The dissolution profiles of the pure drug, marketed formulation and lipid-based solid dispersions formulations are shown in Figures 4. It was observed that the dissolution rate has been increased significantly compared to the pure drug formulation and marketed formulation. Formulation ASD6 has shown the highest drug release in 30 minutes. Improved drug dissolution might be due to the high intrinsic solubility of the drug in the presence of gelucire 50/13 and hydrophilic coat formation of Pearlitol SD200 surrounding the drug.

\section{In Vivo Pharmacokinetic Study}

In vivo studies revealed that the bioavailability of optimized formulation is high compared to pure drug and marketed product. The plasma concentration data were given in table 4. The pharmacokinetic parameters were calculated and listed in table 5. Plasma concentration-time profile is shown in figure 5. It was observed that the oral bioavailability of optimized formulation is increased by 1.11 times and 1.04 times when compared to pure drug and marketed formulation respectively.

\section{CONCLUSION}

In the present study, the potential of lipid-based solid dispersions to improve the bioavailability of water-insoluble drug Azilsartn Medoxomil was investigated. The results showed that saturation solubility, dissolution, and in vivo bioavailability has been increased to a greater extent. Thus lipid-based solid dispersion technology shall be used to improve the bioavailability of poorly water-soluble drugs that will make the dosage form will be cost-effective.

\section{REFERENCES}

1. Lindenberg M, Kopp S, Dressman JB. Classification of orally administered drugs on the World Health Organization Model list of Essential Medicines according to the biopharmaceutics classification system. Eur J Pharm Biopharm 2004;58:265-278

2. Pouton CW. Formulation of poorly water-soluble drugs for oral administration: physicochemical and physiological issues and the lipid formulation classification system. Eur J Pharm Biopharm 2006; 29: 278-287.

3. Sucheta B, Dyandevi M, Mithun VKP, Rajendra DP. Solubility enhancement of antihypertensive agent by solid dispersion technique. Int J Pharm Life Sci 2011; 2(8):970-975

4. Gaurav Subhash K, Bidkar SJ, Dama GY. Formulation \& Evaluation of Ciprofloxacin Solid Dispersion Controlled Release Floating Capsules for Solubility Improvement. Ind J Pharm and Biol Res 2017; 5(3):7-16.

5. Kumar MK, Kohli S, Jain AP. Evaluation of dissolution enhancement of lovaststin by solid dispersion technique. Int J Phar and Life Sci 2011; 2(7):894-898

6. Radhika Reddy P, Manjusha Reddy P, Siva Koti Reddy A. Enhancement of bioavailability of Carbamazepine by solid dispersion technique. Int J Res Pharm Chem 2013; 3(1):54-56

7. Nadia S, Yeakuty M Naz Hasan H. Dissolution Profile of Ibuprofen Solid Dispersion Prepared with Cellulosic Polymers and Sugar by Fusion Method. Saudi J Pharm Sci 2011; 4(1): 31-37.

8. Bhandari KH, Newa M, Kim JA, Yoo BK, Woo JS, Lyoo WS, Lim HT, Choi HG, Yong CS. Preparation, characterization and evaluation of coenzyme Q10 binary solid dispersions for enhanced solubility and dissolution. Biol Pharm Bull 2007; 30: 1171-1176.

9. Craig DQM. The mechanisms of drug release from solid dispersions in water-soluble polymers. Int J Pharm 2002; 231:131-144.

10. Huda NH, Saffoon N, Jhanker YM. Dissolution Enhancement of Ibuprofen Solid Dispersion Prepared with Vinyl Polymers by Fusion Method. Stamford J Pharm Sci 2010; 3(2): 7-11.

11. Laeis, P., Puchler, K., Kirch, W. The pharmacokinetic and metabolic profile of Azilsartan medoxomil limits the risk of clinically relevant drug interaction. J Hyperten 2001; 19: 21-32

12. Nakagomi-Hagihara, R., Nakai, D., Kawai, K., Yoshigae, Y., Tokui, T., Abe, T., Ikeda, T. Oatp1b1, Oatp1b3 and Mrp2 are involved in hepatobiliary transport of Azilsartan, a novel angiotensin II Blocker. Drug Met Disp 2006; 34: 862-869

13. Yamada, A., Maeda, K., Kamiyama, E., Sugiyama, D., Kondo, T., Shiroyanagi, Y., Nakazawa, H., Okano, T., Adachi, M., Schuetz, J. D., Adachi, Y., Hu, Z., Kusuhara, H., Sugiyama, Y. Multiple human isoforms of drug transporters contribute to the hepatic and renal transporter of Azilsartan, a selective antagonist of the angiotensin II AT1-receptor. Drug Met Disp 2007; 35: 2166-2176.

14. Chaitanyaprasad M, Vidyasagar G, Rao KR, Ramanjeneyulu S. Development of RP-HPLC method for estimation of Azilsartan medoxomil in tablet dosage forms. Der Pharm Chem 2011;3:208-12

15. Yohan V, Syed M, Srinivasarao D. Research article on optimization of Azilsartan tablet formulation by 2, 3 factorial design. Int J Res Nanosci 2015;4:188-95. 
Table 1: Composition of Lipid Based Solid Dispersions

\begin{tabular}{lcccccc} 
Name of the Ingredient & ASD & ASD2 & ASD $_{3}$ & ASD $_{4}$ & ASD $_{5}$ & ASD6 \\
Azilsartan Medoxomil API & 40 & 40 & 40 & 40 & 40 & 40 \\
Gelucire 44/13 & 40 & 80 & 120 & -- & -- & -- \\
Gelucire 50/13 & -- & -- & -- & 40 & 80 & 120 \\
Pearlitol SD 200 & 120 & 80 & 40 & 120 & 80 & 40 \\
Total Unit Weight (mg) & 200 & 200 & 200 & 200 & 200 & 200 \\
\hline
\end{tabular}

Table 2: Three way crossover treatment study for the selected formulations

$\begin{array}{lll}\text { Sequence } 1 & \text { Sequence } 2 & \text { Sequence } 3\end{array}$

$\begin{array}{lllll}\text { Group } 1 & \text { ASD6 } & \text { Zilarbi } & \text { APD } \\ \text { Group 2 } & \text { Zilarbi } & \text { APD } & & \\ \text { Group 3 } & \text { APD } & \text { ASD6 } & & \text { ASD3 }\end{array}$

Table 3: Results of Formulations

\begin{tabular}{|c|c|c|c|c|c|c|c|}
\hline \multirow[t]{2}{*}{ Parameters } & \multicolumn{7}{|c|}{ Formulations } \\
\hline & Pure Drug & ASD1 & $\mathrm{ASD}_{2}$ & $\mathrm{ASD}_{3}$ & $\mathrm{ASD}_{4}$ & ASD $_{5}$ & ASD6 \\
\hline Bulk Density & 0.35 & 0.57 & 0.63 & 0.73 & 0.61 & 0.62 & 0.75 \\
\hline Tapped Denisty & 0.66 & 0.62 & 0.74 & 0.77 & 0.66 & 0.68 & 0.84 \\
\hline Angle of Repose (ㅇ) & 38.7 & 23.2 & 17.5 & 17.5 & 17.7 & 19.3 & 19.4 \\
\hline Carr's Index & 47.0 & 8.1 & 14.9 & 5.2 & 7.6 & 8.8 & 10.7 \\
\hline Hausner's Ratio & 1.9 & 1.1 & 1.2 & 1.1 & 1.1 & 1.1 & 1.1 \\
\hline Assay (\%) & 100.1 & 99.2 & 99.6 & $99 \cdot 4$ & 99.6 & $99 \cdot 7$ & 99.8 \\
\hline $\begin{array}{l}\text { Saturation Solubility in } \\
\text { Water }(\mathrm{mg} / \mathrm{mL})\end{array}$ & 0.08 & $15 \cdot 54$ & 32.24 & 45.56 & 22.56 & 48.87 & 65.57 \\
\hline
\end{tabular}

Table 4: Plasma Concentration Profile of Azilsartan Formulations

\begin{tabular}{lccc} 
Time (hrs) & \multicolumn{1}{c}{ Plasma Concentration $(\mathbf{n g} / \mathbf{m L})$} & ASD6 \\
\hline APD & 0.00 & Zilarbi & 0.00 \\
0.5 & 63.73 & 0.00 & 121.08 \\
1 & 179.48 & 86.77 & 272.40 \\
1.5 & 348.21 & 184.60 & 431.91 \\
2 & 581.15 & 374.87 & 696.77 \\
3 & 770.80 & 604.44 & 836.52 \\
4 & 984.01 & 786.57 & 1111.65 \\
6 & 817.38 & 1010.06 & 897.08 \\
8 & 762.13 & 861.11 & 838.14 \\
10 & 717.48 & 784.62 & 783.98 \\
12 & 653.84 & 722.08 & 733.59 \\
18 & 550.19 & 672.74 & 593.96 \\
24 & 446.90 & 554.57 & 497.14 \\
\hline
\end{tabular}


Table 5: Pharmacokinetic Parameters of Azilsartan Formulations

\begin{tabular}{lcccc} 
Parameter & Unit & APD & Zilarbi & ASD6 \\
K & $1 / h$ & 0.033 & 0.031 & 0.033 \\
t1/2 & $\mathrm{H}$ & 20.82 & 22.24 & 20.95 \\
Tmax & $\mathrm{H}$ & 4 & 4 & 4 \\
Cmax & $\mathrm{ng} / \mathrm{ml}$ & 984.01 & 1010.06 & 111.64 \\
AUC o-t & $\mathrm{ng} / \mathrm{ml}{ }^{* h}$ & 14829.55 & 15220.82 & 16467.18 \\
AUC o-inf_obs & $\mathrm{ng} / \mathrm{ml}^{*} \mathrm{~h}$ & 28254.77 & 30079.76 & 31493.47 \\
AUMC o-inf_obs & $\mathrm{ng} / \mathrm{ml}^{*} \mathrm{~h}^{\wedge} \mathbf{2}$ & 894295.40 & 1006141.38 & 1000534.49 \\
MRT o-inf_obs & $\mathrm{H}$ & 31.65 & 33.44 & 31.76 \\
Vz/F_obs & $\mathrm{Ltrs}$ & 0.318 & 0.320 & 0.287 \\
Relative BA with APD & & & & 111.46 \\
Relative BA with Zilarbi & & & & 104.70 \\
\hline
\end{tabular}

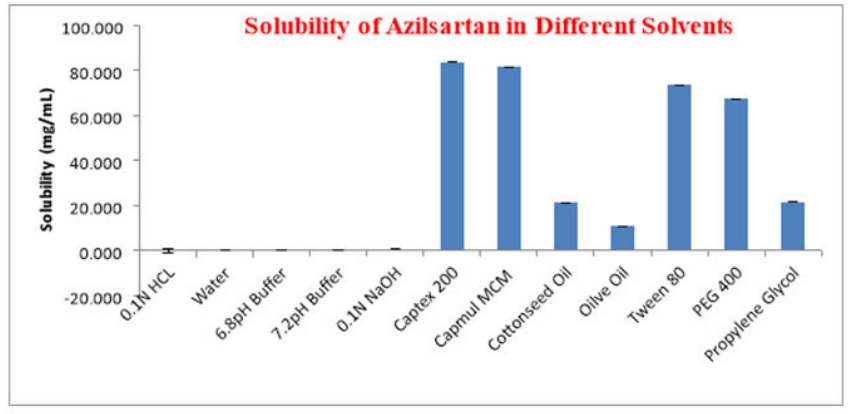

Figure 1: Solubility of Azilsartan in different solvents.

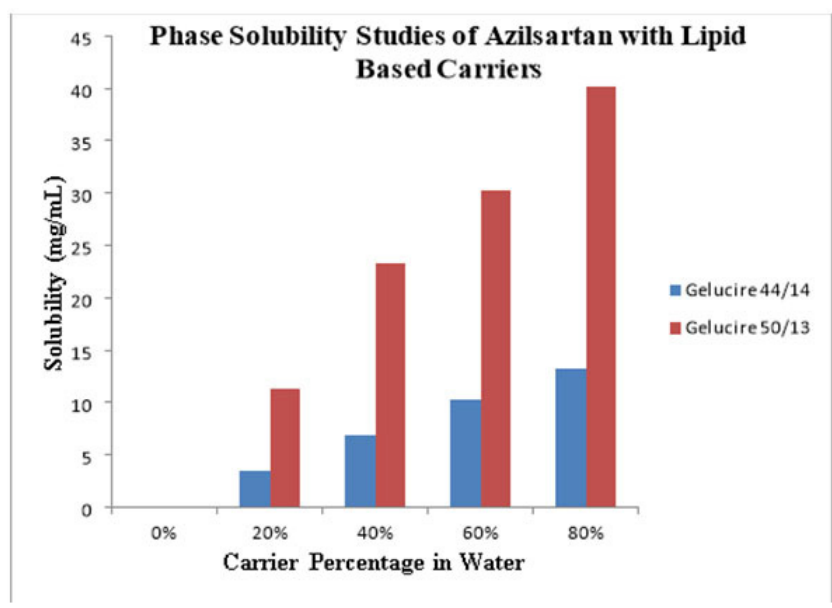

Figure 2: Phase Solubility Study Results of Azilsartan.

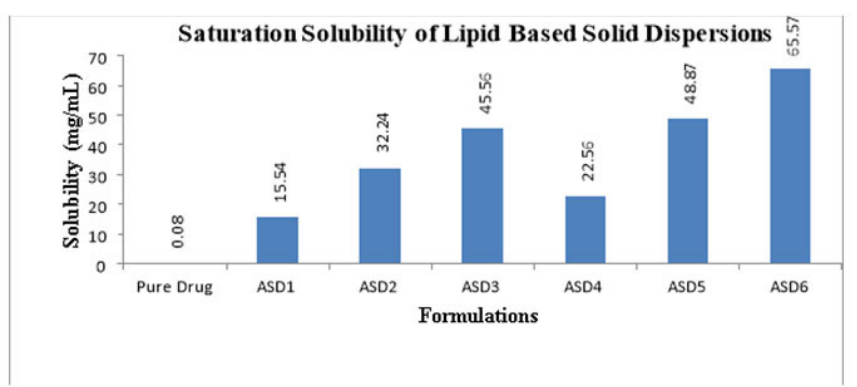

Figure 3: Saturation Solubility results of Lipid based solid dispersions in water.

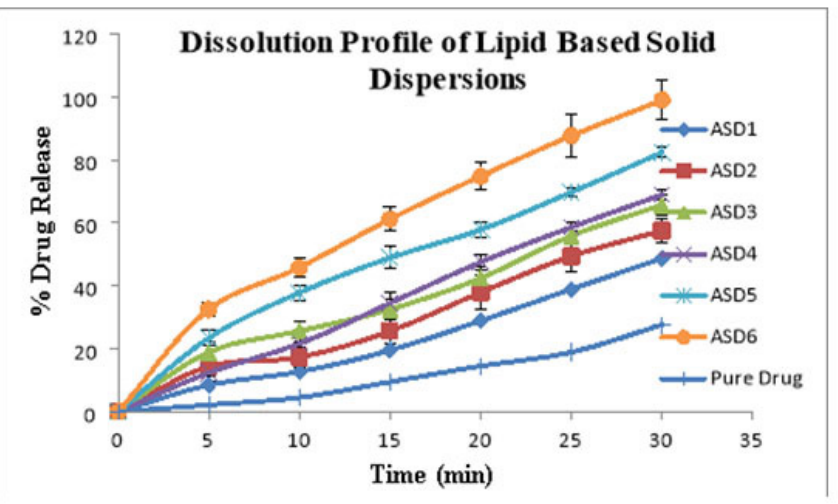

Figure 4: Dissolution Profile of Lipid Based Solid Dispersions.

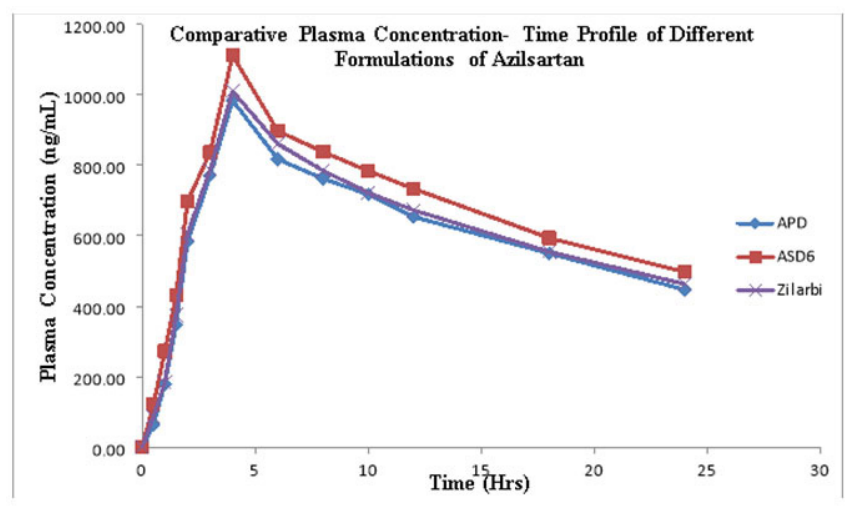

Figure 5: Comparative Plasma Concentration- Time Profile of Different Formulations of Azilsartan. 